\title{
Sea-trial of Optical Ethernet Modems for Underwater Wireless Communications
}

\author{
G. Cossu, A. Sturniolo, A. Messa, S. Grechi, D. Costa, A. Bartolini, D. Scaradozzi, \\ A. Caiti Fellow, IEEE and E. Ciaramella Senior Member, IEEE
}

\begin{abstract}
A new pair of optical wireless modems has been realized, which exploit Visible Light Communication (VLC) to transmit Ethernet signals through water. The modem prototypes are finally tested in sea waters at La Spezia harbor; they successfully transmitted $10 \mathrm{Mbit} / \mathrm{s}$ 10Base-T signals over a up to $10 \mathrm{~m}$, notwithstanding the high turbidity and the strong sunlight. Final tests included the integration with SUNRISE testbed and the use with a moving robot, remotely operated. Commercial components were used to realize the modems; thus, we expect that the key design concepts can be used as a starting point for practical deployment of this technology.
\end{abstract}

Index Terms - underwater communication, visible light communication, Light Emitting Diodes, Wireless communication

\section{INTRODUCTION}

Wireless data transmission usually relies on electromagnetic waves in the radio-frequency (RF) part of the spectrum. However, the underwater environment represents a relevant exception, since water strongly attenuates RF waves [1-4]. Submarine exploration and monitoring is now being extended to greater regions and depths, thanks to the development of autonomous underwater vehicles (AUVs) [5], which allow for an increasing number of tasks. AUVs, however, need communication means to transmit the conveyed information at the control stations on land or ships: although wired transmission might be feasible, wireless communication is by far more practical. However, since RF cannot be exploited, today the submarine communications mostly rely on acoustic waves [6]. Unfortunately, only low-frequency acoustic waves exhibit low attenuation [7], high-frequency waves suffer from strong absorption and mechanical limitations. This strongly limits the available bandwidth of common acoustic modems around few $\mathrm{kHz}$ and hence their bit-rate [8]: high-performance acoustic modems can reach transmission rates up to $35 \mathrm{kbit} / \mathrm{s}$ [9], but common products have a typical rate of few kbit/s [10]. Moreover, the above rates are achieved in optimal channel conditions: the strong variability of the underwater acoustical

G. Cossu, A. Sturniolo, A. Messa and E. Ciaramella are with Scuola Superiore Sant'Anna University, Istituto di Tecnologie della Comunicazione, dell'Informazione e della Percezione (TeCIP), Via Moruzzi 1, 56124 Pisa, Italy. (e-mail: g.cossu@santannapisa.it). A. Bartolini and D. Scaradozzi are with Dipartimento di Ingegneria dell'Informazione, Università Politecnica delle Marche, Via Brecce Bianche, 12, 60131, Ancona, Italy. D. Costa is with Dipartimento di Ingegneria Industriale e Scienze Matematiche, Università communication channel, due to the varying oceanographic conditions, strongly degrades in practice the performance of acoustic modems [11].

While mankind is actively pursuing explorations of the Moon and outer space, around $95 \%$ of the world's oceans and $99 \%$ of the ocean floor still remain unexplored [12]. Recently, the underwater activities are increasing, pushing the research for novel solutions. There is a wide variety of systems deputed at sea-monitoring, ranging from buoys, ships, the aforementioned AUVs, Remotely Operated Vehicles (ROVs) [13-15] and Underwater Sensor Networks (UWSNs) [16]. AUVs currently represent a well-proven technology [17-23]. These vehicles can record high-resolution images, videos, sonar and other data, and transmit them to a central unit or a backbone, without resurfacing, even in very shallow water or even in harbor environment. For this type of data, high bit rate and low latency transmission is usually required. Due to these requirements, the underwater systems are often forced to wired solutions, that impact on device mobility and re-configurability, limiting the possible applications [24].

Combining high-speed and wireless transmission, the Underwater Optical Wireless Communications (UOWC) is a valid alternative, which is rapidly gaining popularity. Recently, the impressive developments in Light Emitting Diodes (LEDs) for lighting purposes made widely available compact devices of low-cost and significant modulation bandwidth. This also opened the way to the terrestrial (indoor) Visible Light Communications (VLC), which use LEDs to transmit wireless signals. VLC recent implementations have shown very high bit rates [25-29]; this gave momentum to the use in underwater environment, as sea-water has a low-loss window in the visible region that can be conveniently exploited by UOWC. Thanks to this, UOWC can offer much higher bit-rates, compared to acoustic modems, although at shorter distances. Depending on bit rate and water attenuation, this technology can reach Mbit/s rates over tens of meters [30-33]. Both Laser Diodes (LDs) or LEDs have been proposed in UOWC systems. The former have much wider bandwidth and higher optical power, but are more expensive compared to LEDs, are prone to misalignment and

Politecnica delle Marche, Via Brecce Bianche, 12, 60131, Ancona, Italy. S. Grechi and A. Caiti are with University of Pisa, Dipartimento di Ingegneria dell'Informazione, Largo Lucio Lazzarino - 56122 Pisa. S. Grechi is now with Honda Racing F1. This work was partially funded by European Union, ICT FP7 funding scheme, Project "SUNRISE", Grant n. 611449 
may require active temperature stabilization [34-36]. First LED-based UOWC systems was able to transmit data at a rate of $5-20 \mathrm{Mbit} / \mathrm{s}$ over ranges up to $100 \mathrm{~m}$ in clear, dark waters (2400 m depth) [37, 38]. Other blue-light LED-based systems (see for instance, without ambition of being exhaustive were later reported (see $[39,40]$ and references therein). A product is also available [41], in two versions: one claimed to deliver 5 Mbit/s at $10 \mathrm{~m}$ range in 'high ambient light' conditions, the other with data rate between 2.5 and $12.5 \mathrm{Mbit} / \mathrm{s}$ at ranges up to $150 \mathrm{~m}$, which is claimed "suitable for moderate to low turbidity dark water (> $200 \mathrm{~m}$ depth or night-time)".

These achievements are quite impressive, nevertheless they share many limitations, in particular in very shallow water and high turbidity conditions. An operational environment with such conditions is that of commercial and military harbors, where AUV-based patrolling and surveying is actively developed [42], with the constraint of not resurfacing in order not to interfere with normal harbor operations.

In recent years, the Authors used blue LEDs and Discrete Multi-Tone to demonstrate $58 \mathrm{Mb} / \mathrm{s}$ in $2.5 \mathrm{~m}$ clear water, in an outdoor pool with a waterproof transmitter (TX) and a receiver (RX), both submerged [43]. The system worked under strong sunlight illumination, which usually poses a severe limitation to the achievable Optical Signal to Noise Ratio (OSNR). Building on this preliminary result, here we present the final design and the at-sea tests of an optical communication system (OptoCOMM), oriented, but not restricted, to operations in daylight harbor conditions, i.e., in presence of both high sunlight disturbance and high water-turbidity.

The system was realized in the framework of the EU-FP7 SUNRISE project [44], and it is thus suitable for integration in the project network of underwater things [45, 46]. The results reported here show that the OptoCOMM UOWC modems provide a reliable 10Base-T IEEE standard bi-directional transmission (10 Mbit/s Ethernet) at a range up to $10 \mathrm{~m}$ in very shallow harbor water and daylight condition. On the application layer side, the system is also fully integrated with the SUNSET software framework of the SUNRISE project, allowing the operation and data transmission both from local and remotely connected working stations (as shown in Fig. 1).

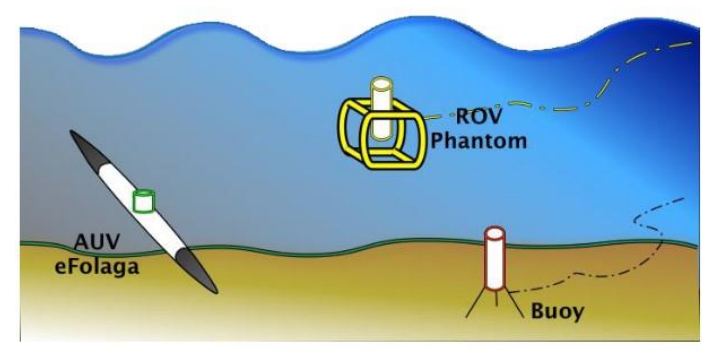

Fig. 1. The OptoCOMM scenario where three optical modems are equipped on a AUV, on a ROV and on a buoy connected to the infrastructure.

In [47], we reported the preliminary results of the early stage of the OptoCOMM development, particularly dealing with testing of the optical part. In this paper, the final version of the modems is described, and the performance in sea-trial tests of the closed system is reported in terms of packet loss, transmission distance, sunlight illumination and water turbidity. A short summary of these tests was recently presented in [48].

This paper is organized as follows. In section II, we describe the key limiting effects sin our UOWC case. In section III, we present the design of the proposed modems and the equipment used for the demonstration. In section IV, we report the results obtained in the lab to finally characterize the optical components and the system performance. In section $\mathrm{V}$, we present the results of the sea-trials measurements performed at La Spezia harbor, namely at NATO's Centre for Maritime Research and Experimentation (CMRE) and at the Italian Navy's Centro di Supporto e Sperimentazione Navale (CSSN) facilities.

\section{UNDERWATER COMMUNICATION CHALLENGES}

In this section, we provide a summary of the main transmission impairments that were considered when designing the modems, i.e. the signal attenuation and the sunlight influence.

\section{A. Optical Loss}

In an UOWC link, three main effects contribute to the signal attenuation: absorption, scattering and beam divergence. The loss coefficients due to the absorption, $a(\lambda)$, and the scattering, $b(\lambda)$, are usually combined in the extinction coefficient $k(\lambda)$ :

$$
k(\lambda)=a(\lambda)+b(\lambda)
$$

As known, water attenuation has a minimum in the visible region [49]. The exact wavelength of the lowest attenuation depends on the specific composition of the sea water and it can be affected by the presence of biological and non-biological elements. In clear and pure waters, the best propagation is attained around the blue-green region $(\sim 40 \mathrm{~nm})$. This value shifts towards longer wavelengths when the turbidity increases [50]. Since our modems are designed to operate also in clear waters, we choose blue LEDs for our TX.

In UOWC, precise alignment and tracking with a moving robot would be very hard to attain, e.g. due to water currents. Therefore, in order to have wide tolerance to misalignment we realized the system with non-negligible beam divergence. Obviously, in addition to scattering and absorption, optical beam divergence also affects the final received power: from the receiver point of view, it may be equivalent as a source of loss. Therefore, a simplified expression can be derived to estimate the received power $\left(P_{\text {opt }}\right)$ vs. the distance $d$, on axis:

$$
P_{o p t}(d) \cong \frac{P_{1}}{d^{2}} \exp (-k(\lambda) d)
$$

where $P_{l}$ is the power received at $1 \mathrm{~m}, d$ is in meters. Indeed, when designing UOWC modems, one must choose the beam divergence, which determines $P_{l}$; a great divergence does not require high accuracy in the modem alignment and can make easier to achieve the link connection. However, a high divergence clearly limits UOWC at long ranges. In our case, the best trade-off is obtained for the non-negligible divergence of the beam of around 20 degrees, which is compatible with our target distance of $10 \mathrm{~m}$ in shallow waters. 
In the real sea, the accurate loss measurement of the optical signal is apparently difficult; moreover, the instantaneous signal loss is also affected by strong variations due to the environment: season, weather, reflections of light at wave surface and water turbidity may change with time, sometimes very quickly. They all affect the measurements, which indeed show both long-term and short-term fluctuations.

In order to measure the value of the extinction coefficient at sea, we can use two alternative methods. First, we can measure $P_{\text {opt }}$ at different distances using the monitoring part of the modems (see later) and then extract $P_{1}$ and $k$ by fitting the various measurements. This technique is more accurate in principle but moving frequently the modems may result unpractical. Mostly, moving the modems takes some time, thus the results can be far from accurate when the short-term variations are very significant.

Alternatively, we can rely on measurements of the turbidity coefficient using a turbidimeter. This second option is by far faster and therefore more practical, although in principle gives only indirect information. In a previous preliminary investigation at the sea test site, we experimentally confirmed that $k$ and Formazin Turbidity Unit (FTU) values are in linear relationship with a slope coefficient of $k / \mathrm{FTU} \approx 0.2[47,51]$. We note that previous independent experimental evidence was reported about a relationship between the light penetration in water and its turbidity, indicating that typical harbor water had $k \sim 0.3 \mathrm{~m}^{-1}$ [52]. These values are all related to a single measurement event. In Fig. 2, we report a histogram of measured turbidity at $1 \mathrm{~m}$ depth, at different times, in the test site location and the same day. As can be expected, the channel conditions change significantly. The modems must be thus designed to work with dynamically varying conditions.

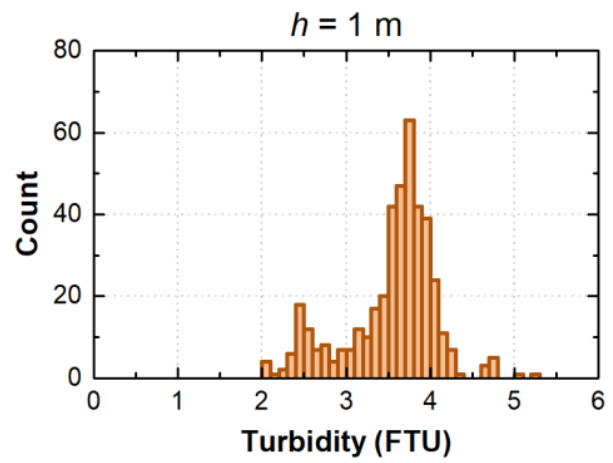

Fig. 2. Histogram of values of turbidity measured at $1 \mathrm{~m}$ depth.

\section{B. Sunlight influence}

Testing UOWC in shallow waters, we face another impairment: in our outdoor conditions, we measured an illuminance level in the order $10^{4}$ lux due to the sunlight [47]. We measured this value as close as possible to the RX, i.e. just above the water surface. Since this measured value is due to sunlight, it is much higher than the typical values of indoor illumination (500 lux), which are common in the other VLC implementations. Even assuming the effect of a band-pass filter at RX, sunlight is so relevant that it produces a photo-current much higher (a factor $\sim 10^{2}$ ) than the signal current. The main effect is the saturation of the RX, which induces penalty [47].

The effect of sunlight is also changing. Sunlight arrive at the $\mathrm{RX}$ directly or because of reflections and scattering in water. At different hours, seasons and weather conditions, the sunlight can be directional, rather than diffused, and can be closer or farther to the transmission axis. The estimated background optical power, integrating the entire spectrum in the visible region, gives a value of $4.5 \mathrm{dBm} / \mathrm{cm}^{2}$ at the water surface, at noon.

Furthermore, the working depth affects the role of ambient light, e.g. shallow harbor waters are far noisier then deep oceans. Even with the observed high-turbidity, we estimate that at our operating depth $(1 \mathrm{~m})$, the direct sunlight is attenuated by a minor fraction (between 1.5 and $3 \mathrm{~dB}$ ). Although these conditions are extremely stressing for the system, it was designed to work with very high levels of background light, even much higher than the signal [47].

\section{OPTICAL MODEM DESIGN}

In this section, we describe the key elements of our final UOWC modems: we present a general description of the modems as a whole, with the optical part, the software design and the watertight containers.

\section{A. Optical Communication part}

The optical section is composed of two parts: the UOWC part constituted by the optical TX and RX, which allow for bidirectional communication, and the monitoring part, which helps in estimating the optical power at the RX. The scheme of the optical modems is shown in Fig. 3.
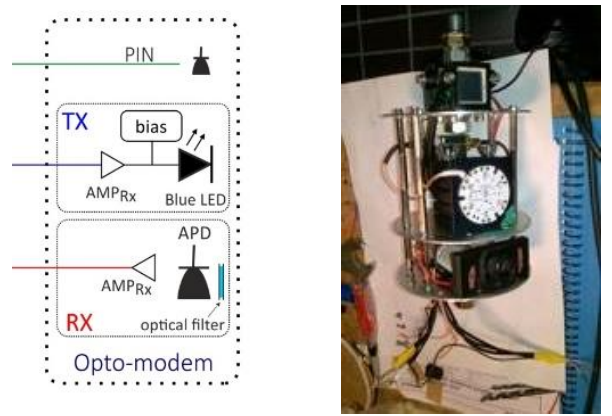

Fig. 3. Scheme of the UOWC modem (left) and the picture of one of the UOWC optical modems (right). The three floors contain (from top to bottom): the monitoring photodiode, the transmitter LEDs and the receiver.

The TX is made by a 7-chips LED array, connected in series, with emission wavelength peaked at $470 \mathrm{~nm}$ (blue light) and 20 $\mathrm{nm}$ optical bandwidth. The LED chips are biased at $21 \mathrm{~V}$ (around $400 \mathrm{~mA}$ ) and give a total luminous power of $40 \mathrm{dBm}$. The electrical signal from the Ethernet interface, after an amplification stage (Amp Tx $_{2} 25 \mathrm{~dB}$ gain), is added by a bias-tee to the dc bias voltage of the LED. In order to increase the optical power at the RX a plastic array of 7 lenses reduces the divergence of each LED, down to around $20^{\circ}$. In the aligned condition, this provides an optical gain around $11 \mathrm{~dB}$.

The RX encompasses a single Avalanche Photo-Diode (APD) module with an active area of $100 \mathrm{~mm}^{2}$, a bandwidth of $11 \mathrm{MHz}$ 
and an integrated transimpedance amplifier (TIA). In front of the APD an optical bandpass filter ( $470 \mathrm{~nm}$ central wavelength, $30 \mathrm{~nm}$ optical bandwidth) is used to reduce the impact of other light sources. We also decrease the FOV of the RX down to around $20 \mathrm{deg}$ [47], to further reduce the received sunlight power detected by the APD, $P_{b k g}$. An example of eye diagram taken at the RX is shown in Fig. 4. We recall that here the transmitted signal, being 10Base-T Ethernet, is Manchesterencoded.

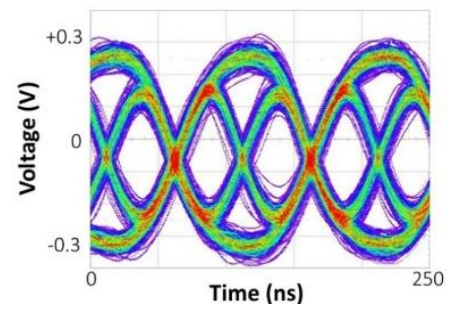

Fig. 4. Typical eye diagram at the RX, taken after the electrical amplifier.

The APD output is amplified by a variable voltage amplifier (Amp $\mathrm{Rx}, 10$ to $40 \mathrm{~dB}$ gain) to produce a signal with the electrical amplitude compliant with the Ethernet interfaces of common PCs. Finally, the monitoring part is made of a simple PIN photodiode; this allows a reasonable estimation of the amount of light arriving at the RX, which includes both signal and background.

\section{B. Electronics and Software}

The on-board modem electronics includes ancillary sensors (e.g., humidity and temperature), power (Lithium Polymer battery), and a Field Programmable Gate Array-based Application Real Time Microcontroller board (ARM/FPGA) for firmware/software installation and overall modem control and management. The selected ARM/FPGA board is the National Instrument (NI) myRIO, composed by an ARM Cortex A9 processor and a Xilinx FPGA, including the NI Linux Real Time Operating Systems, analog and digital connections, and Ethernet ports. In addition, the electronics includes several DC-DC converters, with appropriate analog filtering and insulation to avoid power cross-talk on both data and power lines.

The software developed on the NI myRIO manages the commands received from a user and sends files through the optical layer. Moreover, to make the UOWC modems integrated in the SUNRISE networking structure, we realized the modem interface compliant with the SUNSET Software Defined Communication Stack (SDCS) [53, 54].

The interface with the SUNSET SDCS is described later in this section. In the following the direct software interface with the optical modems is described. The user communicates with the modem via TCP/IP Ethernet protocol in order to change the modem settings, to retrieve information regarding the modem status, and to send and to receive data via optical communication. This is implemented through two main logical layers, one for the User Interface (UI) and another for managing the optical communication. Only one user at time can take advantage of the modem functionalities as commonly adopted in this type of applications, leaving multi-user management to a higher level as offered from the SUNSET SDCS framework The UI is based on ASCII commands; the user, identified as client, needs to connect to the optical modem, server, on two different ports (identified as 1 and 2 in the following). On Port 1 the user sends the commands and receives the corresponding response, on Port 2 the user receives the acknowledgement and the data from an optical link. The two ports interface is motivated by the different responses and the way to process them: on Port 1 the modem replies sequentially to each command sent by the user as confirmation of the reception. Instead, on Port 2 the user receives asynchronous responses. All messages on both ports are started by a Header identifying the message class.

There are four main message classes that can be sent over Port 1:

1. STATUS: request about the physical status of the modem (humidity, temperature, battery power);

2. SET: determines the modem setting for optical transmission (modem identifier, number of attempts to start transmission, number of re-sending attempt for each packet if no acknowledgment is received);

3. GET: retrieves the current modem setting (as established by a previous SET command);

4. DATA: specifies the data to be optically sent to another modem, including modem identifier, acknowledge request (yes/no), data payload size and data payload.

On Port 2 the user receives outcomes regarding an optical transmission with another modem. These outcomes can be ACK (acknowledgement) messages and REC (received data) packets. ACK messages include the coding of errors in transmission/reception ( 0 for no errors), the number of sent packets and the number of received packets. The REC messages include the identifier of the sender nodes, the data payload length and the data payload itself. As for the optical communication protocol, the user is informed about the communication outcome by receiving ACK and REC packets on Port 2 (see the scheme in Fig. 5).

To achieve the maximum throughput and minimize the overhead of each packet, the UOWC is based on Ethernet User Datagram Protocol (UDP). Compared to the TCP, the UDP is not a reliable protocol, is not connection-oriented, and does not guarantee the order of the arriving packets. Despite these missing features, the UDP is the only plausible choice due the physical limitations imposed by the shared means of transmission, the water. However, in order to enhance the reliability of the UDP protocol, a custom header of fixed length was added in each packet and a retransmitting procedure was implemented. The header is chosen to minimize the overhead, but also to include the necessary information for a successful transmission. Each sent packet is identified and ordered by an incremental numeric value; in addition, the header contains the total transmission size and the identification of the sender and receiver modems.

From a higher level integration, including networking in a UWSN with multiple nodes, a specific OptoCOMM driver for the SUNSET SDCS system was implemented relying on the UI messages described previously. This integration includes a 
specific fragmentation module to allow SUNSET SDCS to transmit packets with arbitrary sizes without having to take into account the maximum packet size that a specific modem can transmit [55]. The fragmentation module is transparent to both users and protocol stack. On the TX side, once the fragmentation module receives a packet from the upper layer, it first checks its size. If that exceeds the maximum payload size allowed by the modem in use, it splits the data in smaller packets (chunks) and forwards them to the lower layer(s) of the protocol stack. On the RX side, the fragmentation module reassembles the original packet once all chunks are received. Laboratory testing of the SUNSET SDCS interface were conducted [55], exchanging from remote interfaces via a Virtual Private Network (VPN) data files of size ranging from 11 MBytes to 1.5 GBytes. A similar data exchange was repeated in the experimental test reported in the following.

\section{Watertight containers}

The optical and electronic parts of each of our modems, once completed, were inserted into their watertight containers. Each container is composed by an assembly of two parts: the Body and the Head. Both parts are composed by a cylindrical tube, enclosed between two acetyl neck flanges. The Body tube is made from an aluminum alloy and hosts the electronics. The Head case is a 3-mm wall tube and made from Perspex. Both tubes are bonded to the corresponding flange necks using special sealing adhesive. The upper flange of the Head and the lower flange of the Body are closed by an acetyl blind flange constrained by six bolted screws. A double radial O-ring seal (NBR, $3 \mathrm{~mm}$ Cross-Section, $100 \mathrm{~mm}$ Internal Diameter) was realized between the blind flanges and the corresponding internal surfaces of the necks. The upper neck flange of the Body is connected to the lower neck flange of the Head using six bolted screws. A double axial O-ring seal (NBR, $3 \mathrm{~mm}$ Cross-Section, $90 \mathrm{~mm}$ and $105 \mathrm{~mm}$ Internal Diameter) was realized between the contact surfaces to prevent any water leakage in the modem case joining section.

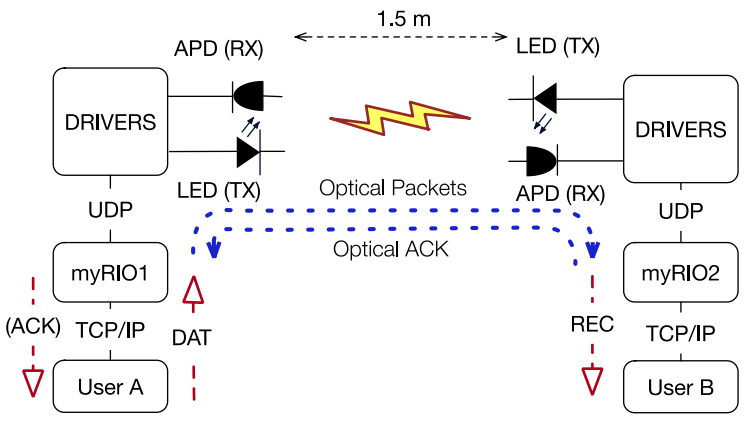

Fig. 5. Scheme of transmission from User A to User B.

\section{FinAl LAB TESTS}

\section{A. Optical section}

Before the final at-sea experiment, exhaustive tests in the lab, spanning various parameters of the UOWC system, were performed. Since in shallow water the main source of noise is the ambient light, we first characterized the RX under strong background light. Using the setup presented in [47], we simulated various environmental conditions, such as different transmission distances and different levels of ambient light, performing transmission measurements with different OSNR.

The goal of these tests was to characterize the effect of the ambient light on the RX performance, varying both the received optical power from the TX $\left(P_{s}\right)$ and $P_{b k g}$. For no background, as expected, the signal amplitude increase as $P_{s}$ increases. When introducing $P_{b k g}$, the electrical signal amplitude initially remains unaffected, but after a saturation value is reached, the APD saturation reduces the signal amplitude, therefore we must increase $P_{s}$ in order to maintain the performance, which result into a power penalty. This will be the most common working condition, because the ambient light in normal conditions is higher than this saturation value [47].

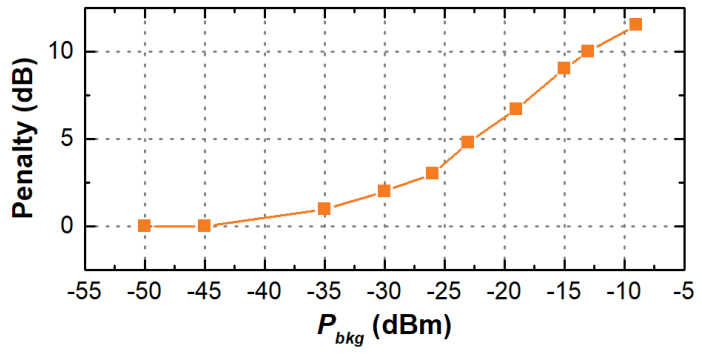

Fig. 6. Power penalty to guarantee a transmission in error-free condition for a varying value of background light.

On the final UOWC modem, we thus measured the penalty introduced by a varying $P_{b k g}$, while still allowing for error-free transmission. In Fig. 6, we report the obtained penalty for each value of $P_{b k}$. As long as $P_{b k g}<-40 \mathrm{dBm}$, the RX sensitivity is around $-39 \mathrm{dBm}$. At higher $P_{b k g}$ values, a relevant penalty arises, which can reach $10 \mathrm{~dB}$ at $P_{b k g} \sim-14 \mathrm{dBm}$, i.e. quite close to our operating conditions. Yet, the link is still working, although with a significant penalty.

These data give a means to predict the behavior of the system under strong illumination. As example, we expect that our UOWC system works even with strong ambient light of $4.5 \mathrm{dBm} / \mathrm{cm}^{2}$ (optical power at the water surface during a sunny day in summer time) when the received signal power $P_{s}$ is at least $-26 \mathrm{dBm}$. Depending on extinction coefficient at our site, different transmission distances can be achieved. For instance, considering a value $k=0.25 \mathrm{~m}^{-1}$ and strong ambient light background as above, a distance of $10 \mathrm{~m}$ can be achieved with $40 \mathrm{dBm}$ power source, which is the optical power by our TX.

\section{B. Containers pressure tests}

In order to test the modem specifications, both the described parts of the hull were tested in a custom pressure chamber available at the CMRE facility, La Spezia. The Body reached a pressure approximately equivalent to $100 \mathrm{~m}$ depth without showing any sign of structural damage and water leak. The Head, being of Perspex, has proved to reach $50 \mathrm{~m}$ depth by maintaining its fully integrity and the required watertight properties. Failure depth for the Head pressure hull was measured at about $60 \mathrm{~m}$ depth. Before the sea experiments, the modem pressure hull has been tested fully assembled in order to verify the axial seal in the connection section. The tests and measurements were conducted at the same CMRE facility, La 
Spezia. Both pressure hulls proved to reach $20 \mathrm{~m}$ depth without showing any sign of water leakage.

\section{SEA TRIALS}

The final sea-trials of the project were performed at Centre for Maritime Research and Experimentation (CMRE) in La Spezia (Italy), at the same facility and site where preliminary tests occurred [47]. A high-view picture of the testbed is reported in Fig. 7 (left). As can be seen, the experimental area is located in the middle of two piers, within the commercial part of La Spezia harbor, and it is partially closed. Thus, the water turbidity at the site is much higher than in open sea.

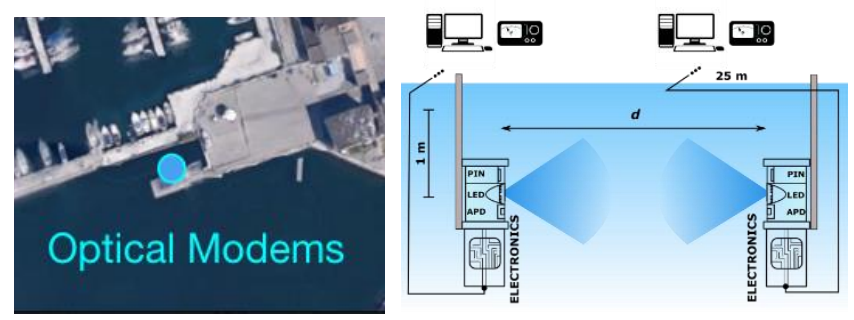

Fig. 7. Left: satellite view of the trial site. Right: experimental setup of the transmission tests with fixed modems, which are connected by cable to common PCs (in a container on the pier).

The setup used during the experiments is sketched in Fig. 7 (right). The modems were clamped to the two posts. Both modems were submerged so that the optical layers (at the top of each) were at $1 \mathrm{~m}$ depth. A picture of the modems before submersion is shown in Fig. 8. One of the modems (M1) was fastened to a pole and connected via a $25 \mathrm{~m}$ long Ethernet cable to a PC. This submarine cable transmits the TCP/IP commands (via power line communication) from the user interface but also supplies $24 \mathrm{~V}$ to the modem. The monitoring equipment and the PCs were inside a container on the pier. The second modem (M2) acted as a mobile node; thus, it could be moved by a forklift at increasing distances from M1. M2 was also connected to a PC via a similar cable. Since the modems are designed to operate with Ethernet 10Base-T, we had no access to their physical layer (e.g. we could not measure eye diagrams, Bit Error Ratio etc.). We had only accessible the Ethernet link, which could be monitored by sending and receiving Ethernet packets from the PCs, thus measuring the packet loss.

During the tests, we also measured the turbidity at the site as a function of water depth by means of a turbidimeter (Ocean Seven 305 Plus by Idronaut), in terms of Formazin Turbidity Unit (FTU). Measurements of turbidity were taken at roughly three hours intervals starting at 9.00 a.m. As an example, in Fig. 9 , we report the turbidity values measured of at different depths and times during the day of trial. We see that at this particular site turbidity increases with depth (sea floor is sandy and is around at around $5 \mathrm{~m}$ depth). Furthermore, measurements taken at different times in the same day show quite different behavior.

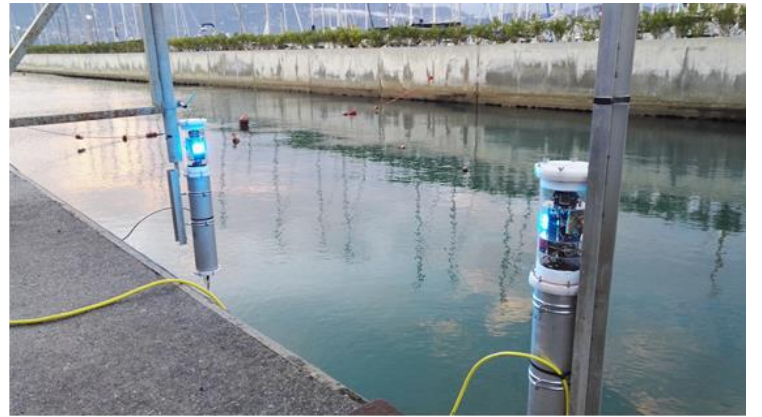

Fig. 8 Picture of the modems before submersion at $d=2.5 \mathrm{~m}$; poles and connection cables can be clearly seen.

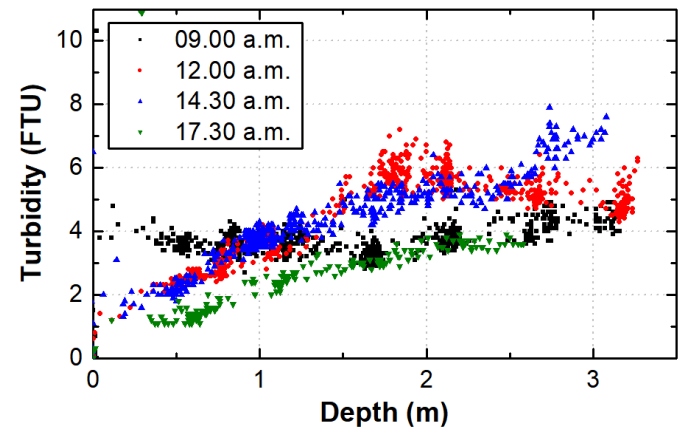

Fig. 9 Measured turbidity values as a function of depth at different hours in the same day. Note the fluctuation of turbidity within the day but also within the same instrumentation cast.

\section{A. Transmission with fixed modems}

During the days of the trials, we faced variable ambient conditions, both in water turbidity and in background light, which both had to be continuously monitored. Particularly, turbidity was unusually high, and this had a strong impact on the signal attenuation.

The communication experiments consisted in repeated file transfers from one PC to the other through the underwater link. In these tests we first measured the packet loss as a function of the distance between the modems (from 2.5 to $10 \mathrm{~m}$ ), with the modems submerged and fixed on the two poles that were shown in Fig. 8. In order simulate a real scenario we transferred two different file types as test, i.e., a photo and a video, having size of 3.4 and 14.4 Mbytes, respectively.

Our measurements started at the shortest distance of $2.5 \mathrm{~m}$ : here, not surprisingly, we obtained the best transmission conditions, although we had $P_{b k g} \sim P_{s}$ even with high turbidity and sunlight effect was indeed around $-11.5 \mathrm{dBm}$. Notwithstanding that, the packet loss was always found to be zero. The following set of measurements were taken at $5 \mathrm{~m}$ distance. Here the received signal was lower $\left(P_{s} \sim-22 \mathrm{dBm}\right)$ and now the ambient light was higher than the signal. Still the observed packet-loss was zero, in all cases. Further increasing the distance to $7.5 \mathrm{~m}$, makes the signal more than 10 times lower than the background at the RX, which gives a minimum $(<1 \%)$ loss of data at $7.5 \mathrm{~m}$. Increasing the distance beyond this value, the received optical signal power was so low that the monitoring system was not able to measure precisely its value. Beyond this distance, we could only estimate the received optical power exploiting the values collected at shorter distance, adapting the data to distance and turbidity level (see Fig. 10 right). 


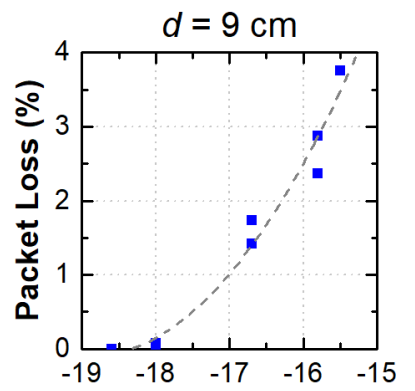

Sunlight power (dBm)

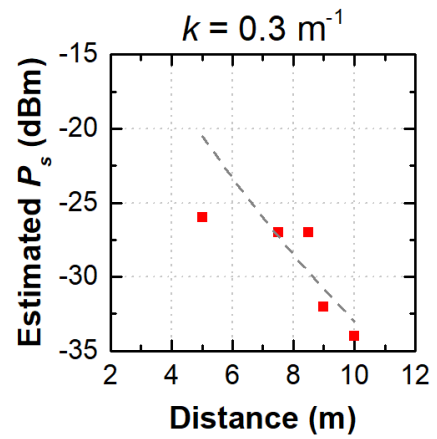

Fig. 10 Left: observed packet loss as function of sunlight background illumination, taken at $9 \mathrm{~m}$ distance. Right: Summary of estimated signal power as function of distance, fitted with attenuation profile assuming $k=0.3 \mathrm{~m}^{-1}$.

TABLE I PACKET TRANSMISSION RESULTS AT $10 \mathrm{M}$ DISTANCE

\begin{tabular}{|c|c|c|c|}
\hline Packets Sent & $\boldsymbol{P}_{\boldsymbol{b k g} \boldsymbol{g}}(\mathbf{d B m})$ & Packets Lost & Packet Loss (\%) \\
\hline \hline 3408 & -19.6 & 14 & 0.1 \\
\hline 3408 & -19.6 & 35 & 0.2 \\
\hline 3408 & -19.6 & 19 & 0.13 \\
\hline 14376 & -19.6 & 9 & 0.06 \\
\hline 14376 & -20.4 & 5 & 0.03 \\
\hline 14376 & -20.4 & 1 & 0.01 \\
\hline 14376 & -21 & 0 & 0 \\
\hline 14376 & -21 & 0 & 0 \\
\hline
\end{tabular}

TABLE II MEASURED PARAMETERS AND PACKET LOSS AT DIFFERENT DISTANCES

\begin{tabular}{|c|c|c|c|c|}
\hline Distance (m) & $\boldsymbol{P}_{\text {bkg }}(\mathbf{d B m})$ & $\boldsymbol{P}_{\boldsymbol{s}}(\mathbf{d B m})$ & Turbidity (FTU) & Packet Loss (\%) \\
\hline \hline 2.5 & -11.5 & -11.5 & $3-4$ & 0 \\
\hline 5.0 & -10.4 & -22.4 & $3-4$ & 0 \\
\hline 7.5 & -10 & -27.0 & $3-4$ & 0.26 \\
\hline 10 & -21 & -34.0 & $2-3$ & 0 \\
\hline
\end{tabular}

Finally, we transmitted the Ethernet signal over the $10 \mathrm{~m}$ distance, which is the maximum expected distance of the OptoCOMM project. At this distance the received optical power was estimated to be around $-34 \mathrm{dBm}$. We transferred several photos and video files in error-free condition, but only when the background optical power reached $-21 \mathrm{dBm}$, a condition that we encountered at sunset. The measured value of turbidity in this last test fluctuated between $2-3$ FTU. The results are reported in Table I, where we indicate with $P_{b k g}$ the optical power of the background light estimated with the PIN photodiodes. A complete summary of the packet loss results at different distances is presented in Table II.

\section{B. Measurements with a Remotely Operated Vehicle}

One of the optical modems was also installed on a small Remotely Operated Vehicle (ROV), VideoRay Pro4, to test transmission with a moving modem. Fig. 11 shows the optical modem clamped under the ROV, which was teleoperated from the pier with a console via umbilical cable. The ROV was exclusively steered in manual mode, without resorting to the vehicle heading and depth control. Fig. 12 illustrates a moment of the experiment. File transmission from the ROV to the fixed node at a maximum range of $5 \mathrm{~m}$ was performed, in which the file transferred was the 3.5 MB image. The communication was successfully completed: the file was repeatedly transferred in its integrity and the acknowledgment packet was received, over a total transmission time of 10 minutes. Since the ROV was manually maneuvered to keep line of sight with among the modems, the vehicle was always in movement during the transmission. Thanks to the chosen beam divergence, the communication quality was not affected. Such accomplishment proves also the robustness of the optical modems and the feasibility of optical communication during an operation at sea.

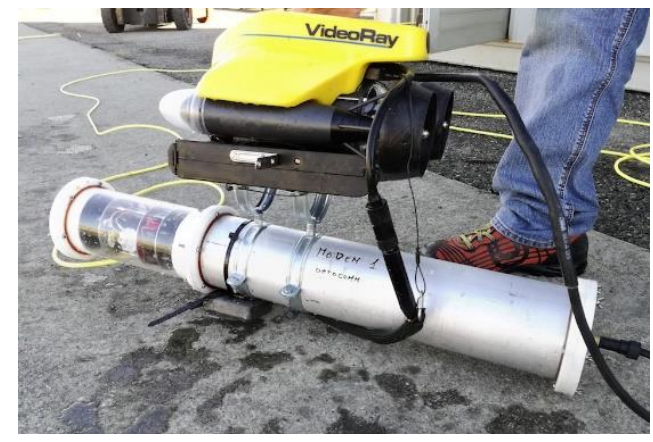

Fig. 11 UOWC modem equipped under the ROV for mobile transmission.

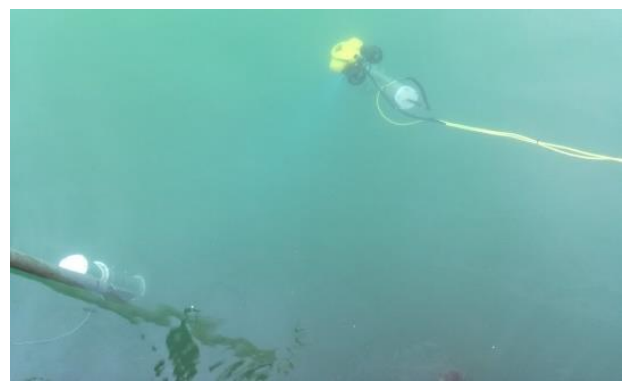

Fig. 12 Picture of the experiment with a moving modem installed on the ROV. The modems are at $1 \mathrm{~m}$ depth, and a faint blue light can be seen from the ROV modem. The signal is barely visible due to the high turbidity.

\section{SUNSET integration}

Finally, the modems were tested in the fixed configuration when integrated in the SUNSET framework. The integration was one of the main goals of our project and it soundly demonstrated that the modems, in their present form, are already suitable to deploy an Internet of Underwater Things.

We note that the modems had been previously tested in the laboratory tests with SUNSET SDCS interface; the same test was repeated during the sea trial. The two optical modems M1 and M2 were connected via cables to two instances of SUNSET SDCS running on two different PCs. A Virtual Private Network (VPN) connection between the University of Rome and the harbor of La Spezia allowed the users to access to SUNSET SDCS, whose fragmentation module was used to transmit large files overcoming the constraints of the maximum packet size of the optical modem.

Several files with different sizes, ranging from 11 MBytes to 1.5 GBytes, were optically transmitted. The maximum payload size for each packet was 5 Mbytes; the greater files were fragmented by the SUNSET SDCS fragmentation module at the TX and then reassembled by the RX. 


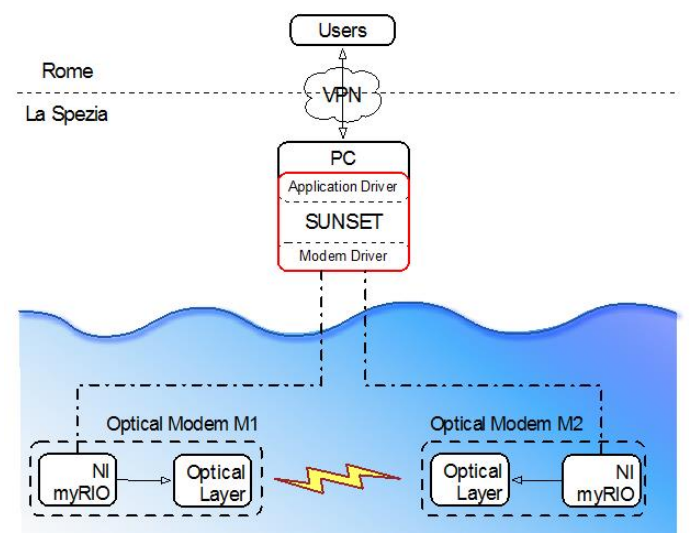

Fig. 13 Top: Block-diagram of the remote connection through the SUNSET framework; Bottom: results on data transmission through the SUNSET SDCS both in transmission and in reception.

Fig. 13 illustrates the setup during the experiments. We transmitted files of various sizes from 11 to 1.5 GBytes (these values do not include the different headers used by SUNSET SDCS for each layer of the protocol stack). All files were successfully transmitted among the optical modems and sent back to another SUNSET remote station. The test was performed with the modems at a range of $5 \mathrm{~m}$ one from the other, with background noise power (due to sunlight illumination) of approximately $-11 \mathrm{dBm}$ (average through the experimental time). It is worth noting that, while the optical modems operated always at $10 \mathrm{Mb} / \mathrm{s}$ rate, the overall SUNSET network rate was on average $4 \mathrm{Mb} / \mathrm{s}$ (see Table III), mainly due to delay and latency introduced by the VPN.

The results were very similar to what had been previously achieved within the lab testing. This confirmed the achievement of the integration between the optical modems and the SUNSET framework also in at sea operative scenarios.

TABLE III PACKET TRANSMISSION RESULTS IN SUNSET INTEGRATION

\begin{tabular}{|c|c|c|c|}
\hline File Size & \# Chunks & Transmission rate [Mb/s] & Packet Loss \\
\hline \hline $11 \mathrm{MB}$ & 3 & 4.70 & 0 \\
\hline $46 \mathrm{MB}$ & 10 & 4.15 & 0 \\
\hline $106 \mathrm{MB}$ & 22 & 4.27 & 0 \\
\hline $354 \mathrm{MB}$ & 72 & 3.99 & 0 \\
\hline $500 \mathrm{MB}$ & 101 & 4.30 & 0 \\
\hline $750 \mathrm{MB}$ & 151 & 4.27 & 0 \\
\hline $1.25 \mathrm{~GB}$ & 251 & 4.31 & 0 \\
\hline $1.50 \mathrm{~GB}$ & 301 & 4.26 & 0 \\
\hline
\end{tabular}

\section{CONCLUSIONS}

We designed and demonstrated a novel UOWC system, useful for practical marine experiments and fully compatible with 10Base-T Ethernet transmission $(10 \mathrm{Mbit} / \mathrm{s}$ with a Manchester-coded signal). The modem includes a photonic section, an electronic part and a software part for integration within the project SUNRISE networking architecture.

The complete final version of the modems was tested at sea, in harbor condition (high turbidity) and with sunlight disturbance. During the test, the measured turbidity level at the transmission depth was between 2.5 and 5 FTU, much higher than previously experienced at the same location [47]. Performance in the final test was assessed by measuring the packet loss as a function of distance, while monitoring water turbidity and background optical noise. The system was proven error-free or with negligible packet loss up to a range of $7.5 \mathrm{~m}$ in full daylight and very high turbidity. At sunset, with decrease of the optical noise to $-21 \mathrm{dBm}$, error-free transmission at $10 \mathrm{~m}$ was achieved. In both cases (full daylight, sunset) the performance achieved improves with that reported by other systems in comparable conditions.

The transmission between a fixed and a mobile node was carried out successfully with one of the modems installed on a ROV. This accomplishment assessed the good robustness of the adopted technology, based on diverging beams, and the feasibility in the employment of such technology in at sea operative scenarios. Finally, the integration between the SUNSET SDCS and the optical modems during the experiment was also successfully tested to guarantee interfacing and networking possibilities.

While the test of the system reported here is indeed in harbor, the design requirements are not restricted to shallow water. Therefore, our developed modems reached the right trade-off that could guarantee improved performance (w.r.t. the state-ofthe-art) in shallow waters without degrading the expected performance in deep, dark and clear waters. While open sea, deep-water transmission was not tested, the laboratory calibrations and the estimated relation between turbidity and extinction coefficient allow to predict an achievable transmission range of at least $40 \mathrm{~m}$ in deep, dark waters.

\section{ACKNOWLEDGMENTS}

The authors gratefully acknowledge the personnel of CMRE and of Naval Support and Experimentation Centre of Italian Navy (CSSN) for their support during the experiments, mostly the technical support from P. Guerrini and J. Alves.

\section{REFERENCES}

[1] S. Sendra et al., "Study of the Optimum Frequency at 2.4GHz ISM Band for Underwater Wireless Ad Hoc Communications," 2012.

[2] S. I. Inácio et al., "Dipole antenna for underwater radio communications," in Underwater Communications and Networking Conference (UComms), 2016 IEEE Third, pp. 1-5.

[3] E. Jimenez et al., "Investigation on radio wave propagation in shallow seawater: Simulations and measurements," in 3rd Underwater Communications and Networking Conference, Ucomms 2016, 2016, pp. $1-5$.

[4] U. M. Qureshi et al., "RF Path and Absorption Loss Estimation for Underwater Wireless Sensor Networks in Different Water Environments," Sensors, vol. 16, no. 6, p. 890, Jun. 2016.

[5] D. Scaradozzi et al., "BCF swimming locomotion for autonomous underwater robots : a review and a novel solution to improve control and e ffi ciency," Ocean Engineering, vol. 130, no. October 2016, pp. 437453, 2017.

[6] J. H. Cui et al., "The challenges of building scalable mobile underwater wireless sensor networks for aquatic applications," IEEE Network, vol. 20, no. 3, pp. 12-18, May 2006.

[7] P. G. Sasi, B. Rao, and S. Member, "Path loss analysis of Underwater communication systems," IEEE Technology Students' Symposium, pp. 65-70, Jan. 2011.

[8] B. Li et al., "Further results on high-rate MIMO-OFDM underwater acoustic communications," in OCEANS 2008, 2008, pp. 1-6.

[9] H. Kaushal and G. Kaddoum, "Underwater Optical Wireless Communication," IEEE Access, vol. 4, pp. 1518-1547, 2016.

[10] S. Sendra et al., "Underwater Acoustic Modems," IEEE Sensors Journal, vol. 16, no. 11, pp. 4063-4071, Jun. 2016. 
[11] A. Caiti, E. Crisostomi, and A. Munafò, "Physical Characterization of Acoustic Communication Channel Properties in Underwater Mobile Sensor Networks," in S-CUBE, Springer, 2010, pp. 111-126.

[12] O. S. Board and N. R. Council, Exploration of the Seas: Voyage into the Unknown. National Academies Press, 2003.

[13] L. Sorbi et al., "Robotic tools and techniques for improving research in an underwater delicate environment," Marine Technology Society Journal, vol. 49 , no. 5 , pp. 6-17, 2015.

[14] D. Scaradozzi, G. Conte, and L. Sorbi, "Assisted guidance system for Micro ROV in underwater data gathering missions.," in 2012 20th Mediterranean Conference on Control and Automation, MED 2012 Conference Proceedings, 2012, pp. 1373-1378.

[15] G. Conte et al., "ROS multi-agent structure for autonomous surface vehicles," in MTS/IEEE OCEANS 2015 - Genova: Discovering Sustainable Ocean Energy for a New World, 2015.

[16] K. Alam, T. Ray, and S. G. Anavatti, "A brief taxonomy of autonomous underwater vehicle design literature," Ocean Engineering, vol. 88, pp. 627-630, 2014.

[17] D. Scaradozzi et al., "Innovative technology for studying growth areas of Posidonia oceanica," in 2009 IEEE Workshop on Environmental, Energy, and Structural Monitoring Systems, EESMS 2009 - Proceedings, 2009, pp. $71-75$.

[18] S.-K. Jeong et al., "Design and control of high speed unmanned underwater glider," International Journal of Precision Engineering and Manufacturing-Green Technology, vol. 3, no. 3, pp. 273-279, Jul. 2016.

[19] T. Battista et al., "A Dynamic Model for Underwater Vehicle Maneuvering Near a Free Surface," IFAC-PapersOnLine, vol. 49, no. 23, pp. 68-73, Jan. 2016.

[20] M. Kojima et al., "AUV IRSAS for submarine hydrothermal deposits exploration," in 2016 IEEE/OES Autonomous Underwater Vehicles (AUV), pp. 161-164.

[21] S. D. Ling et al., "Stereo-imaging AUV detects trends in sea urchin abundance on deep overgrazed reefs," Limnology and Oceanography: Methods, vol. 14, no. 5, pp. 293-304, May 2016

[22] A. Munafò et al., "Autonomous underwater vehicle teams for adaptive ocean sampling: A data-driven approach," Ocean Dynamics, vol. 61, no. 11, pp. 1981-1994, Nov. 2011.

[23] T. B. Curtin and J. G. Bellingham, "Progress toward autonomous ocean sampling networks," Deep-Sea Research Part II: Topical Studies in Oceanography, vol. 56, no. 3-5, pp. 62-67, Feb. 2009.

[24] D. Scaradozzi et al., "Tools and techniques for underwater archaeological sites documentation," in Oceans - San Diego 2013, 2013, pp. 1-6.

[25] S. Arnon, "Underwater optical wireless communication network," Optical Engineering, vol. 49, no. 1, pp. 15001-15006, 2010.

[26] G. Cossu, R. Corsini, and E. Ciaramella, "High-Speed Bi-directional Optical Wireless System in Non-Directed Line-of-Sight Configuration," Journal of Lightwave Technology, vol. 32, no. 10, pp. 2035-2040, May 2014.

[27] G. Cossu et al., "5.6 Gbit/s downlink and $1.5 \mathrm{Gbit} / \mathrm{s}$ uplink optical wireless transmission at indoor distances $(\geq 1.5 \mathrm{~m})$," in Optical Communication (ECOC), 2014 European Conference on, pp. 1-3.

[28] V. Jungnickel et al., "A European view on the next generation optical wireless communication standard," in Standards for Communications and Networking (CSCN), 2015 IEEE Conference on, pp. 106-111.

[29] M. Uysal et al., Optical Wireless Communications: An Emerging Technology. Springer, 2016.

[30] J. W. Giles and I. N. Bankman, "Underwater optical communications systems. Part 2: basic design considerations," in MILCOM 2005-2005 IEEE Military Communications Conference, pp. 1700-1705.

[31] A. Boucouvalas et al., "Underwater Optical Wireless Communications with Optical Amplification and Spatial Diversity," IEEE Photonics Technology Letters, 2016.

[32] B. M. Cochenour, L. J. Mullen, and A. E. Laux, "Characterization of the beam-spread function for underwater wireless optical communications links," IEEE Journal of Oceanic Engineering, vol. 33, no. 4, pp. 513-521, Oct. 2008.

[33] S. Tang, Y. Dong, and X. Zhang, "Impulse response modeling for underwater wireless optical communication links," IEEE transactions on communications, vol. 62, no. 1, pp. 226-234, 2014.

[34] K. Nakamura, I. Mizukoshi, and M. Hanawa, "Optical wireless transmission of $405 \mathrm{~nm}, 145 \mathrm{Gbit} / \mathrm{s}$ optical IM/DD-OFDM signals through a 48 m underwater channel," Optics Express, vol. 23, no. 2, p. 1558, Jan. 2015.

[35] H. M. Oubei et al., "4.8 Gbit/s 16-QAM-OFDM transmission based on compact 450-nm laser for underwater wireless optical communication,"
Optics Express, vol. 23, no. 18, p. 23302, Sep. 2015.

[36] A. S. Fletcher et al., "A narrow-beam undersea laser communications field demonstration," in OCEANS 2016 MTS/IEEE Monterey, OCE 2016, 2016, pp. 1-5.

[37] N. Farr et al., "Optical modem technology for seafloor observatories," in OCEANS 2006, 2006, pp. 1-6.

[38] C. Pontbriand et al., "Wireless data harvesting using the AUV Sentry and WHOI optical modem," OCEANS 2015 - MTS/IEEE Washington, no. August, pp. 1-6, Oct. 2015.

[39] J. Xu et al., "OFDM-based broadband underwater wireless optical communication system using a compact blue LED," Optics Communications, vol. 369, pp. 100-105, Jun. 2016.

[40] P. Leon et al., "A new underwater optical modem based on highly sensitive Silicon Photomultipliers," in OCEANS 2017 - Aberdeen, 2017, pp. 1-6.

[41] Sonardyne, BlueComm, [Online]. Available: https://www.sonardyne.com/product/bluecomm-underwater-opticalcommunication-system. [Accessed: 12/03/2017].

[42] S. Reed, J. Wood, and C. Haworth, "The detection and disposal of IED devices within harbor regions using AUVs, smart ROVs and data processing/fusion technology," in 2010 International Waterside Security Conference, WSS 2010, 2010, pp. 1-7.

[43] G. Cossu et al., "Experimental demonstration of high speed underwater visible light communications," in Proceedings of the 2013 2nd International Workshop on Optical Wireless Communications, IWOW 2013, 2013, pp. 11-15.

[44] SUNRISE, [Online]. Available: http://www.fp7-sunrise.eu/. [Accessed: $01 / 01 / 2017]$.

[45] A. Caiti et al., "OptoCOMM : introducing a new optical underwater wireless communication modem," 2016 IEEE Third Underwater Communications and Networking Conference (UComms), pp. 2-6, Aug. 2016.

[46] A. Bartolini et al., "OptoCOMM: Development and experimentation of a new optical wireless underwater modem," in OCEANS 2016 MTS/IEEE Monterey, OCE 2016, 2016, pp. 1-5.

[47] G. Cossu et al., "Full-Fledged 10Base-T Ethernet Underwater Optical Wireless Communication System," IEEE Journal on Selected Areas in Communications, pp. 1-1, 2017.

[48] G. Cossu et al., "Sea-trial of an Ethernet-based Underwater VLC Communication System," in Optical Fiber Communication Conference, 2018, p. Tu2I.2.

[49] S. Q. Duntley, "Light in the Sea," Journal of the Optical Society of America, vol. 53, no. 2, p. 214, Feb. 1963.

[50] N. G. Jerlov, Marine optics, vol. 14. Elsevier, 1976.

[51] L. Ulrik and K. Bundgaard, "Studies of the relationship between suspended sediment concentration and light attenuation," in $B O O K O F$ ABSTRACTS, 2015.

[52] W. A. Swenson, Influence of turbidity on fish abundance in western Lake Superior. Environmental Protection Agency, Office of Research and Development, Environmental Research Laboratory, 1978.

[53] C. Petrioli et al., "The SUNSET framework for simulation, emulation and at-sea testing of underwater wireless sensor networks," Ad Hoc Networks, vol. 34, pp. 224-238, Nov. 2015.

[54] V. Di Valerio et al., "A self-adaptive protocol stack for Underwater Wireless Sensor Networks," in OCEANS 2016 - Shanghai, 2016, pp. 1-8.

[55] A. Caiti et al., "OptoCOMM and SUNSET to enable large data offloading in Underwater Wireless Sensor Networks," in OCEANS 2016 MTS/IEEE Monterey, 2016, pp. 1-5. 